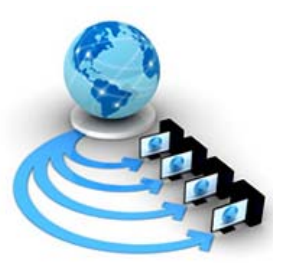

Volume 8, No. 9, November-December 2017

International Journal of Advanced Research in Computer Science

RESEARCH PAPER

\title{
IMPLEMENTATION OF SMART PARKING MANAGEMENT SYSTEM USING APACHE TOMCAT SERVER
}

\author{
M Anila ${ }^{1}$, Raswitha Bandi ${ }^{2}$, N Shirisha ${ }^{1}$, B Durga $\operatorname{Sri}^{2}$ \\ ${ }^{1}$ Department of Computer Science and Engineering, \\ MLR Institute of Technology, Hyderabad, India \\ ${ }^{2}$ Department of Information Technology, \\ MLR Institute of Technology,Hyderabad, India
}

\begin{abstract}
Parking Management System is implemented to manage occupancy of parking slots andallow customers to find and reserve available parking place and to inquire on the vacant slotsbefore vehicle arriving at parking. Parking management system is implemented so that user can book the parking slots in internetbefore arriving to the parking place. The parking management system will encourage customersto book parking slots in online and make the parking process a hassle-free experience.
\end{abstract}

Keywords: parking,TOKEN, Register, Admin, Apache manager.

\section{INTRODUCTION}

Parking Management system is a web based Application to the events of customers. In thissystem, customer can register in the application and get user id and password. Administratorwill manage all the events adding, deleting and updating the slots.Existing system is very difficult to manage and it is a time consuming process. Moremanual work is needed. We have to face high-level risk in maintain the existed system. In orderto overcome all these problems the project is developed from manual procedure to automate.This project has been developed in order to overcome the difficulties encountered while usingthe existing system [1-2].

Parking Management System is developed to support the customers and also themanagement of parking with a flexible and Hassle-free experience. The system enables thecustomer to make registrations and reservations in online [3-5]. Manually the customer has to check the available slot and wait until the slot is free or theysearch for any alternative like any side of roads. This wills a problem for Traffic. To avoid thisproblem Parking Management System is useful for both the customers and the management.In this system all the events are managed by the Admin only [6]. Admin adds, delete and update theinformation in the application. The user or customer login with his login-id and password thenchecks for availability of slots. If the customer has selected a particular slot in a particular stallthen he/she must register by giving the required details. Upon successful registration thecustomer can simply go to that parking place and park the vehicle thereby creating a hasslefreeexperience.

\section{II.PURPOSE \& SCOPE}

The main purpose of Parking Management System is to reduce the manual work andwaiting time of the customer. The customer simply registers in the site and after registration hethen login into the system and he/she check for the available slots. Then the customer reserves the slot by giving the details like name, address, date and time.After completing the reservation the customer simply went to that parking stall and parks thevehicle thereby creating a hassle free experience [7].

As it is automated system it can perform the complete process through online.

\section{A. Existing System}

Exiting system is very difficult to manage parking system because all the events are handledmanually. There are many facts in parking management system like giving place to parkcustomer vehicles in any company is done in Parking Area where TOKEN system is used. It is very Time Consuming andthe customer wants to wait until the slot is free or parking in anysides of Roads [8-10].

\section{A. Drawbacks:}

It is very Time Consuming process.

Manually the users check where the slot is free and park the vehicle with a lot ofphysical work.

Manually the parking team must provide the Token generation and then theyelect the vehicles to park and number of human power is needed.

The Customer has to wait until the slot is free or they search for any alternativelike any side of roads. This wills a problem for Traffic.

There will be some Security issues may arise with this system.

To reach within the time with this existing system is very difficult.

B. Proposed System: 
In this proposed system we are trying to automate the manual process.

In this system the customer should be able to check the parking spaceavailability by specifying the desired date and time using a client device such asWeb browser.

The website will allow customers to reserve a time slot in which to park days, weeks, or even months ahead of time and the system will store this informationand properly arrange parking reservations to maximize the number ofreservations that can be made.

Upon successful reservation, the customer is issued a reservation confirmationnumber.

By this customer can book the parking slots before parking the vehicle.

Advantages:

As this system is automated system, it reduces the manual power.

This system provides Flexibility for reserving the parking slots effectivelywithout any manual power.

There will be no waiting time for the customers.

Using this system there will be no problem with the fines by keeping in the sideroads thereby saving our Money.

\section{III.MODULE DESCRIPTION}

A. Admin:

In this module all the events related to slots and parking events will be maintained and prepared by Admin. Admin can also add, delete and update the slots available for customers. He checks for the registered users and slot booked users. Admin is responsible for maintaining all the events related to this parking management system.

B. Functionalities:

- Admin can add the new slots to the customers.

- Admin can update the slots.

- Admin checks the registered users and the slot booked users.

C. Customer:

In this module, information about all the events will be maintained by the customer belonging to the particular slot. He can register with the application and get user login-id and password. After login with the user id and password the customer checks the vacant slots available and book that selected slot.

C. Functionalities:

- Customer registers with the application.

- Customer login with the login-id and password.

- Customer checks the vacant slots book the selected slot.

D. Data Dictionary

\section{IV.DATABASE:}

We are having 5 Database tables.

- Admin table

- User login table

- User registration table

- Slot parking table

- Booked slots info table

A. Admin table Description:

The Admin table is used for maintaining the Admin login_id and password.

\begin{tabular}{|l|l|l|l|}
\hline Field & Description & $\begin{array}{l}\text { Data } \\
\text { Type }\end{array}$ & Constraints \\
\hline $\begin{array}{l}\text { Admin_name } \\
\text { Admin } \\
\text { password }\end{array}$ & $\begin{array}{l}\text { Admin } \\
\text { name }\end{array}$ & $\begin{array}{l}\text { Varchar } \\
(20)\end{array}$ & Not Null \\
Password & $\begin{array}{l}\text { Varchar } \\
(20)\end{array}$ & \\
\hline
\end{tabular}

B. User login table Description:

\begin{tabular}{|l|l|l|l|}
\hline Field & $\begin{array}{l}\text { Descripti } \\
\text { on }\end{array}$ & Data Type & $\begin{array}{l}\text { Constraint } \\
\text { s }\end{array}$ \\
\hline $\begin{array}{l}\text { Username } \\
\begin{array}{l}\text { User } \\
\text { password }\end{array}\end{array}$ & $\begin{array}{l}\text { User name } \\
\text { Password }\end{array}$ & $\begin{array}{l}\text { Varchar(30 } \\
\text { Varchar(30 } \\
\text { ) }\end{array}$ & $\begin{array}{l}\text { Not Null } \\
\text { Not Null }\end{array}$ \\
\hline
\end{tabular}

C. User registration Table description:

\begin{tabular}{|l|l|l|l|}
\hline Field & Description & Data Type & $\begin{array}{l}\text { Constrain } \\
\text { ts }\end{array}$ \\
\hline Username & Username & Varchar2(1 & Not Null \\
Password & Password & $0)$ & Not Null \\
Designation & Designation & Varchar2(1 & Not Null \\
Date_of_birth & Date_of_birth & $0)$ & Not Null \\
Gender & Gender & Varchar2(1 & Not Null \\
Email_id & Email_id & $0)$ & Not Null \\
Mobile_numb & Mobile_numb & Varchar2(1 & Not Null \\
er & er & $0)$ & Not Null \\
Address & Address & Varchar & Not Null \\
Vehicle_numb & Vehicle_numb & $(10)$ & \\
er & er & Varchar2(1 & \\
& & $0)$ & \\
& & Number(10 & \\
& & ) & \\
& & Varchar2(3 & \\
& & $0)$ & \\
& & Varchar2(1 & \\
& & $0)$ &
\end{tabular}

D. Slot parking table Description:

\begin{tabular}{|l|l|l|l|}
\hline Field & Description & Data Type & Constraints \\
\hline Stall1 & Stall1 & Varchar(10) & Not Null \\
Slot1 & Slot1 & Varchar(10) & Not Null \\
Slot2 & Slot2 & Varchar(10) & Not Null \\
Slot3 & Slot3 & Varchar(10) & Not Null \\
Slot4 & Slot4 & Varchar(10) & Not Null \\
\hline
\end{tabular}




\begin{tabular}{|l|l|l|l|}
\hline Slot5 & Slot5 & Varchar(10) & Not Null \\
Slot6 & Slot6 & Varchar(10) & Not Null \\
Slot7 & Slot7 & Varchar(10) & Not Null \\
Slot8 & Slot8 & Varchar(10) & Not Null \\
Slot9 & Slot9 & Varchar(10) & Not Null \\
Slot10 & Slot10 & Varchar(10) & Not Null \\
\hline
\end{tabular}

\begin{tabular}{|l|l|l|l|}
\hline Field & Description & Data Type & Constraints \\
\hline Username & Username & Varchar(10) & Not Null \\
Stall & Stall & Varchar(10) & Not Null \\
number & number & Varchar(10) & Not Null \\
Slot & Slot & Varchar(10) & Not Null \\
Vehicle & Vehicle & Varchar(10) & Not Null \\
number & number & Varchar(10) & Not Null \\
Date & Date & Varchar2(10) & Not Null \\
Time & Time & Varchar2(10) & Not Null \\
Time & Time period & & \\
period & Amount & & \\
Amount & & & \\
\hline
\end{tabular}

\section{TESTING SCENARIOS}

A. Administrator:

\begin{tabular}{|c|c|c|c|c|}
\hline $\begin{array}{l}\text { S.N } \\
\text { O }\end{array}$ & $\begin{array}{l}\text { Text } \\
\text { case }\end{array}$ & $\begin{array}{l}\text { Expected } \\
\text { value }\end{array}$ & $\begin{array}{l}\text { Actual } \\
\text { value }\end{array}$ & Result \\
\hline 1 & $\begin{array}{l}\text { Enter } \\
\text { user } \\
\text { name } \\
\text { and } \\
\text { passwor } \\
\text { d }\end{array}$ & True & True & Success \\
\hline 2 & $\begin{array}{l}\text { Admin } \\
\text { entered } \\
\text { wrong } \\
\text { passwor } \\
\text { d }\end{array}$ & $\begin{array}{l}\text { Invalid } \\
\text { password }\end{array}$ & $\begin{array}{l}\text { Login } \\
\text { fail }\end{array}$ & Success \\
\hline 3 & $\begin{array}{l}\text { Invalid } \\
\text { user } \\
\text { name }\end{array}$ & $\begin{array}{l}\text { Invalid } \\
\text { user } \\
\text { name }\end{array}$ & $\begin{array}{l}\text { Login } \\
\text { fail }\end{array}$ & Success \\
\hline 4 & $\begin{array}{l}\text { If any } \\
\text { field is } \\
\text { not } \\
\text { entered } \\
\text { while } \\
\text { login }\end{array}$ & $\begin{array}{l}\text { Login } \\
\text { success }\end{array}$ & $\begin{array}{l}\text { Error } \\
\text { will be } \\
\text { occur } \\
\text { indicatin } \\
\mathrm{g} \text { that } \\
\text { enter } \\
\text { specified } \\
\text { field. }\end{array}$ & Failure \\
\hline 5 & $\begin{array}{l}\text { Date } \\
\text { may } \\
\text { specifie } \\
\text { d } \\
\text { incorrec } \\
\text { tly }\end{array}$ & $\begin{array}{l}\text { Login } \\
\text { success }\end{array}$ & $\begin{array}{l}\text { Redirect } \\
\text { to date } \\
\text { field }\end{array}$ & Failure \\
\hline 6 & $\begin{array}{l}\text { Without } \\
\text { entering } \\
\text { passwor } \\
\text { d }\end{array}$ & $\begin{array}{l}\text { Login } \\
\text { success }\end{array}$ & $\begin{array}{l}\text { Enter } \\
\text { password }\end{array}$ & Failure \\
\hline 7 & $\begin{array}{l}\text { Register } \\
\text { ed Users }\end{array}$ & $\begin{array}{l}\text { Displays } \\
\text { the } \\
\text { number }\end{array}$ & $\begin{array}{l}\text { Details } \\
\text { of } \\
\text { registere }\end{array}$ & success \\
\hline
\end{tabular}

\begin{tabular}{|l|l|l|l|l|}
\hline & & $\begin{array}{l}\text { of users } \\
\text { registered }\end{array}$ & $\begin{array}{l}\text { d users } \\
\text { will be } \\
\text { shown in } \\
\text { a table. }\end{array}$ & \\
\hline 8 & $\begin{array}{l}\text { Update } \\
\text { Slots }\end{array}$ & $\begin{array}{l}\text { Vacated } \\
\text { slots of } \\
\text { users can } \\
\text { be } \\
\text { updated. } \\
\text { Update } \\
\text { Successf } \\
\text { ul }\end{array}$ & $\begin{array}{l}\text { Update } \\
\text { successfu } \\
1\end{array}$ & Success \\
\hline 9 & $\begin{array}{l}\text { Slot } \\
\text { booked } \\
\text { users }\end{array}$ & $\begin{array}{l}\text { Displays } \\
\text { the } \\
\text { number } \\
\text { of users } \\
\text { booked } \\
\text { the slots. }\end{array}$ & $\begin{array}{l}\text { Details } \\
\text { of slot } \\
\text { booked } \\
\text { users can } \\
\text { be } \\
\text { displayed } \\
\text { in a } \\
\text { table. }\end{array}$ & \\
\hline
\end{tabular}

\begin{tabular}{|c|c|c|c|c|}
\hline $\begin{array}{l}\text { S.N } \\
\text { O }\end{array}$ & Text case & $\begin{array}{l}\text { Expected } \\
\text { value }\end{array}$ & $\begin{array}{l}\text { Actual } \\
\text { value }\end{array}$ & Result \\
\hline 1 & $\begin{array}{l}\text { Registratio } \\
\mathrm{n}\end{array}$ & $\begin{array}{l}\text { Registratio } \\
\mathrm{n} \\
\text { Successful }\end{array}$ & $\begin{array}{l}\text { Registratio } \\
\mathrm{n} \\
\text { Successful }\end{array}$ & success \\
\hline 2 & $\begin{array}{l}\text { Enter user } \\
\text { name and } \\
\text { password }\end{array}$ & True & True & $\begin{array}{l}\text { Succes } \\
\mathrm{S}\end{array}$ \\
\hline 3 & $\begin{array}{l}\text { User } \\
\text { entered } \\
\text { wrong } \\
\text { password }\end{array}$ & $\begin{array}{l}\text { Invalid } \\
\text { password }\end{array}$ & Login fail & $\begin{array}{l}\text { Succes } \\
\mathrm{s}\end{array}$ \\
\hline 4 & $\begin{array}{l}\text { Invalid user } \\
\text { name }\end{array}$ & $\begin{array}{l}\text { Invalid user } \\
\text { name }\end{array}$ & Login fail & $\begin{array}{l}\text { Succes } \\
\mathrm{S}\end{array}$ \\
\hline 5 & $\begin{array}{l}\text { If any field } \\
\text { is not } \\
\text { entered } \\
\text { while } \\
\text { registration }\end{array}$ & $\begin{array}{l}\text { Registratio } \\
\text { n success }\end{array}$ & $\begin{array}{l}\text { Error will } \\
\text { be occur } \\
\text { indicating } \\
\text { that enter } \\
\text { specified } \\
\text { field. }\end{array}$ & Failure \\
\hline 6 & $\begin{array}{l}\text { Date may } \\
\text { specified } \\
\text { incorrectly }\end{array}$ & $\begin{array}{l}\text { Registratio } \\
n \text { success }\end{array}$ & $\begin{array}{l}\text { Redirect to } \\
\text { date field }\end{array}$ & Failure \\
\hline 7 & $\begin{array}{l}\text { Without } \\
\text { entering } \\
\text { password }\end{array}$ & $\begin{array}{l}\text { Login } \\
\text { success }\end{array}$ & $\begin{array}{l}\text { Enter } \\
\text { password }\end{array}$ & Failure \\
\hline 8 & Select slot & $\begin{array}{l}\text { If vacant } \\
\text { available } \\
\text { book the } \\
\text { slot }\end{array}$ & $\begin{array}{l}\text { Slot booked } \\
\text { successfull } \\
\mathrm{y}\end{array}$ & $\begin{array}{l}\text { Succes } \\
\mathrm{S}\end{array}$ \\
\hline 9 & Select slot & $\begin{array}{l}\text { Booking } \\
\text { the slot that } \\
\text { is already } \\
\text { booked }\end{array}$ & $\begin{array}{l}\text { Your } \\
\text { selected } \\
\text { slot is } \\
\text { booked by } \\
\text { some other } \\
\text { person }\end{array}$ & Fail \\
\hline
\end{tabular}




\section{HOME PAGE}

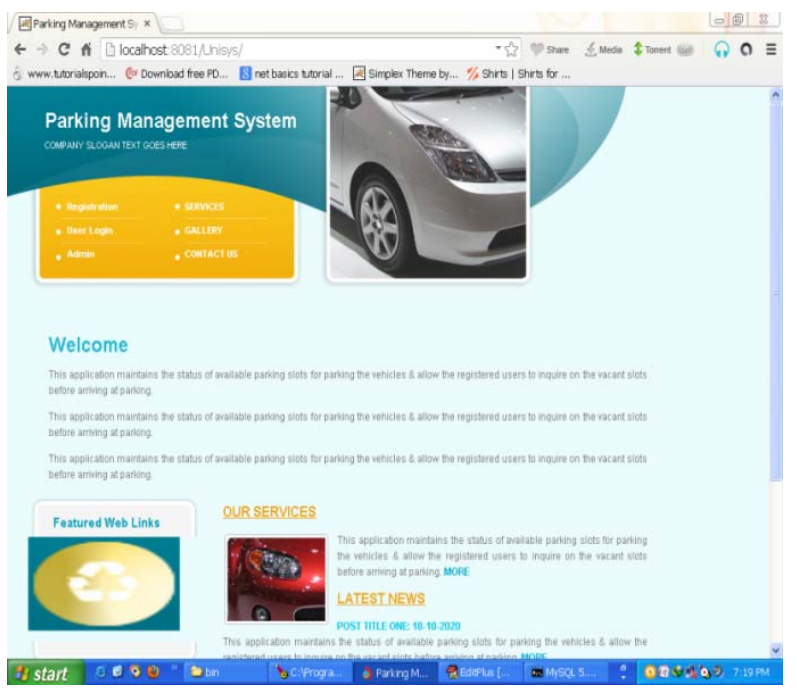

D. ADMIN LOGIN PAGE

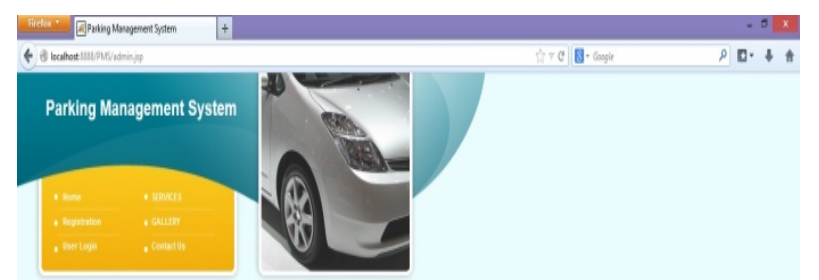

Welcome to Registration Page
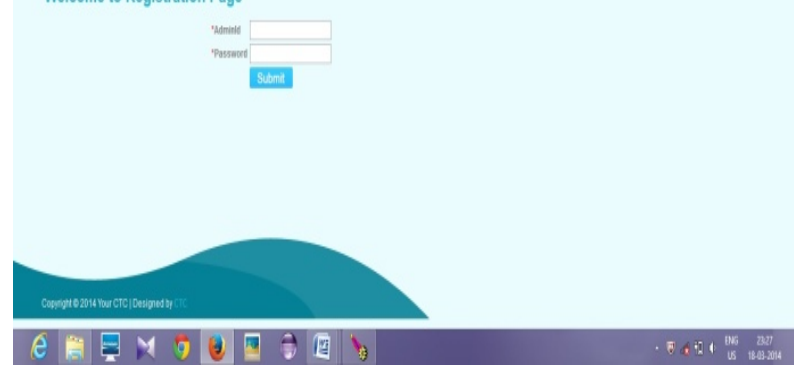

\section{E. REGISTERED USERS}
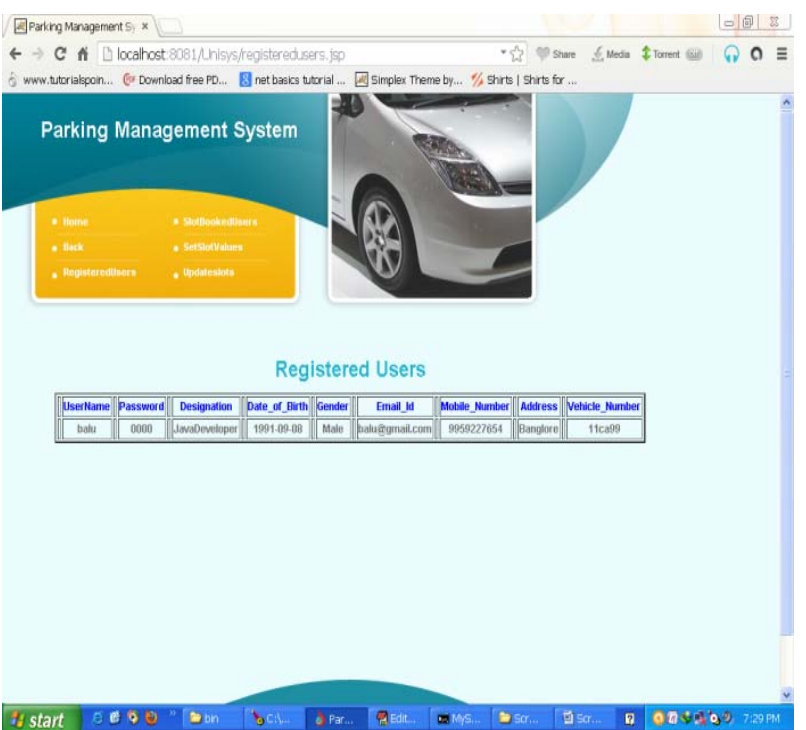

\section{F. SLOT BOOKED USERS}

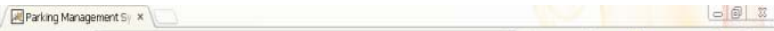

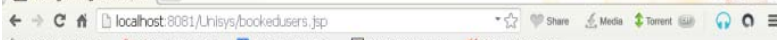

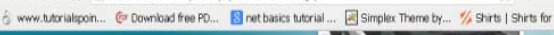

Parking Management System

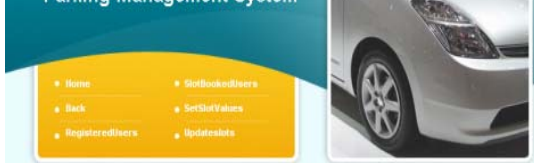

Slot Booked Users
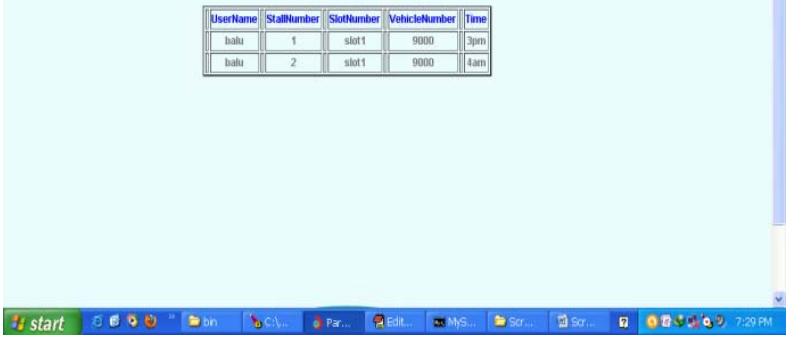

G. SET SLOT VALUES

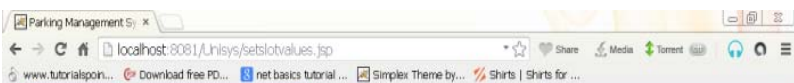

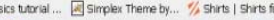

Welcome to User LoginPage
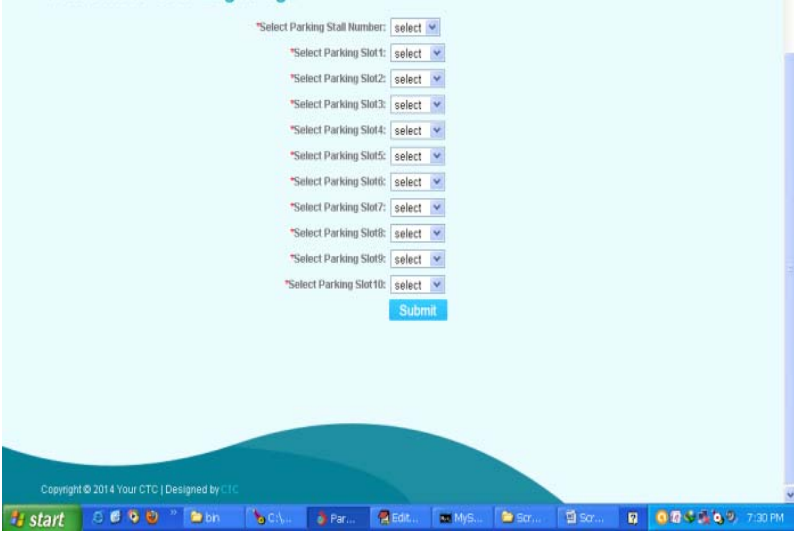

\section{H. CHECK VACANT SLOTS}

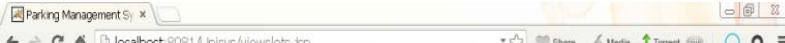

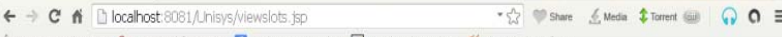

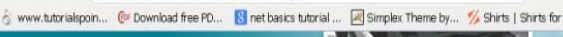

Parking Management System
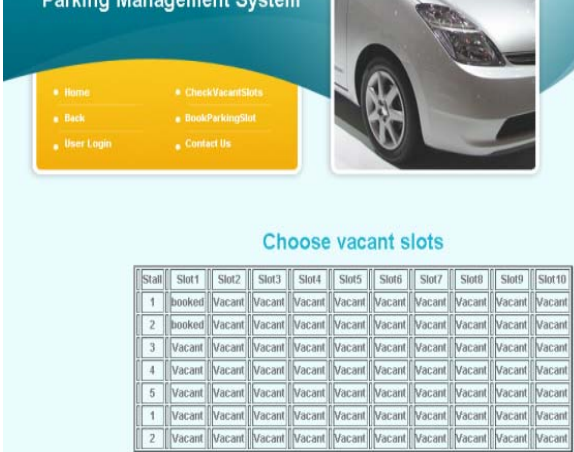

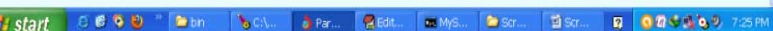




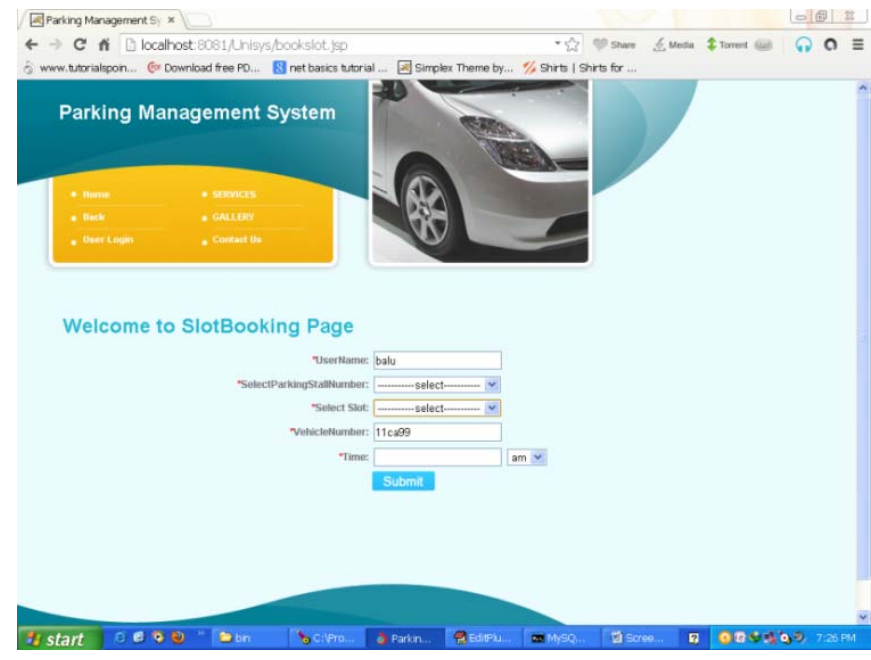

\section{USER MANUAL}

In order to use this Application, we need to install the following software's.

- Mysql database

- $\quad$ Apache Tomcat Server 7.0

After installing these software's we need to deploy the project in the Tomcat server.

\section{Deployment Procedure:}

- Open the location of the Tomcat server.

Apache Software FoundatioTamcat 7.0 Web-apps $\rightarrow$

- Deploy your project in the web-apps folder.

- Start the tomcat server.

- Open mysql command line and connect to the Car Parking Database.

- Open the web-browser and connect to the server by using the port number.(http://localhost:8080)

- Login in to the server by user name and the password.

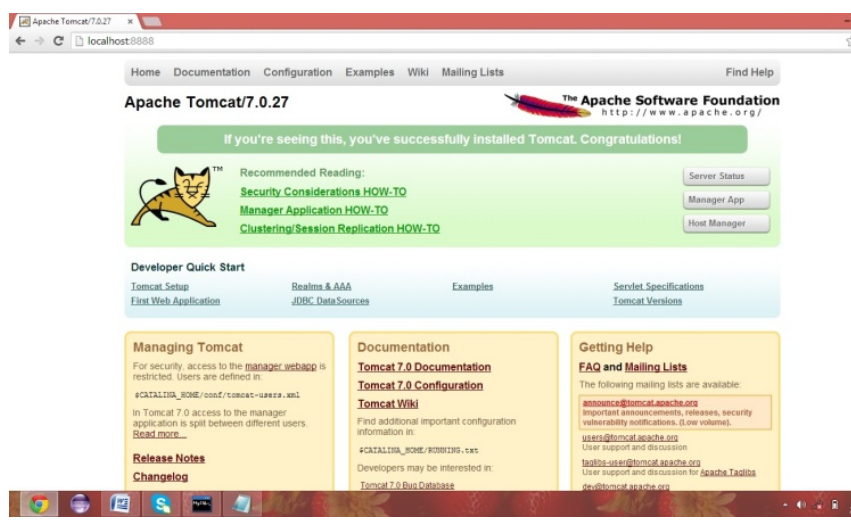

- Click on 'manager App'.

- Click on your project "Parking Management System'. You will get the Home page.

J. Home Page:

The Home page consists of the following Fields.
- Registration

- User login

- Admin

- Services

- Gallery

- Contact us

These are the following events and operations that can be performed by the Admin and the user.

\section{CONCLUSION:}

This paper presents the implementation of a smart parking management system. In this work, we have developed a web application to know the web availability of different parking slots in the respective areas. To implement this, we have used Apache Tomcat server using database SQL server. This technology can be used in the Society viz. Shopping malls, Theatres etc. This system will encourage customers to make parking slots in online and make parking process a Hassle free experience. In future, this application flexibility can be improved by sending the registered id to the user as a text message or e-mail and alerts about the slot information.

\section{REFERENCES}

[1] Prof. A. D. Potgantwar, A. H. Wad, P. P. Pandit, S. Kumar, "RFID BASED VEHICLE IDENTIFICATION SYSTEM AND ACCESS CONTROL INTO PARKING", Patent No.: 1790/MUM/2013A, 05,2013.

[2] Ms. AsmitaJondhale, Ms. Gautami Das, Prof. SamadhanSonavane, "OCR \& RFID Based Vehicle Identification \& Parking Allocation System", IEEE Paper No. 538, vol. 1, pp. 1-5, May. 2015.

[3] Mr. Amrut Ram Selokar, Prof. Shweta Jain, "Automatic Number Plat Recognition System using A Fast StrokeBased Method", IEEE TRANSACTIONS ON MULTIMEDIA, vol. 1, no. 7, pp. 1-5, APRIL 2014, ISSN 2347-3622.

[4] Vandini Sharma, Prakash Mathpal, Akanksha Kaushik, "Automatic license plate recognition using optical character recognition and template matching on yellow color license plate", vol. 3, no. 5, pp. 1-7, May 2014, ISSN 2319-8753.

[5] Lei Xie, Yafeng Yin, Athanasios V. Vasilakos, Sanglu Lu, Managing RFID Data: Challenges Opportunities and Solutions IEEE Paper No.: 06757191, vol. 16, no. 3, pp. 1294-1311, THIRD QUARTER 2014.

[6] Roy Want, "An Introduction to RFID Technology", JanuaryMarch 2006.

[7] Markus Friedrich, Prokop Jehlicka, Johannes Schlaich, "AUTOMATIC NUMBER PLATE RECOGNITION FOR THE OBSERVANCE OF TRAVEL BEHAVIOUR", 8th International Conference on Survey Methods in Transport, May 25-31, 2008.

[8] Christos Nikolaos, E. AnagnostopoulosIoannis, E. AnagnostopoulosVassili, LoumosEleftherios, A Kayafas, "License Plate-Recognition Algorithm for Intelligent Transportation System Applications", vol. 7, no. 3, SEPTEMBER 2006.

[9] C.Y. Suen, R. Plamondon, A. Tappert, A. Thomassen, J.R. Ward, K Yamamoto, "Future Challenges in Handwriting and Computer Applications", vol. 9, no. 4, 2005.

[10] Ron Weinstein, "RFID: Technical Overview and its application to the enterprise", May 2005. 